\title{
Research of Three-Dimensional Measurement Model Base on BI-TELECENTRIC System
}

\author{
Chao Chen ${ }^{1,}$, Ziyang $\mathrm{Xu}{ }^{1, b}$ \\ ${ }^{1}$ School of Electric and Information, South China University of Technology, \\ Guangzhou, Guangdong ,China \\ aeecc@mail.scut.edu.cn, bbeyond-yang@foxmail.com
}

\begin{abstract}
Keywords: industrial measurement; three-dimensional measurement; Bi-Telecentric; three-dimensional reconstruction

Abstract. Combined with recently-developed Bi-Telecentric system, a new three-dimensional measurement model is proposed in this paper based on existing measurement technology, which reduces the calculation of three-dimensional reconstruction. Then, an experimental platform is built to verify the reliability and accuracy of this measurement system. The experiment results show that the measurement accuracy can still reach $0.1 \mathrm{~mm}$ even in a comparatively large measurement field of view.
\end{abstract}

\section{Introduction}

In recent years, three-dimensional measurement is widely used in medical testing, machinery manufacturing, aerospace and other fields. Meanwhile, many new three-dimensional measurement methods and theories are developed[1]. A superfast 3D measuring method based on structured light was proposed by Song Zhong, Purdue University[2]. Zhang group introduced a measuring method based on binocular vision that obtaining character point by Harris process[3]. Quan group measured the key dimensions of workpiece through the contour feature points that was extracted by the Canny operator and polygonal approximation method[4]. In general, there are masses of computations in camera calibration process and feature matching process for the traditional three-dimensional measurement methods.

With the development of modern industry, the industrial measurement is going to require higher precision for three-dimensional measuring technique[5]. The advantage of Bi-Telecentric system that no perspective error, high-resolution, large depth of field, with near-zero distortion, fully fit the demand of industrial measurement [6] .So, in this paper, three-dimensional measurement models based on Bi-Telecentric optical system are going to be discussed, which can theoretically quickly complete a high precision measurement.

\section{Measurement System}

In this paper, the hardware system, as shown in Fig. 1, consists of a line-light source, telecentric cameras, image sensors, testing objects, rotating platform, calibration object, computer, etc. After the camera and rotation-axis are calibrated, adjust the line-light source to the testing object. The camera takes pictures against the highlight whenever the rotating platform rotated by a certain angle. The world coordinates of points in the photographed highlight can be figured out by a series of algorithms. By driving the testing objects at least one round on the rotation platform, its three-dimensional measurement will be finished.

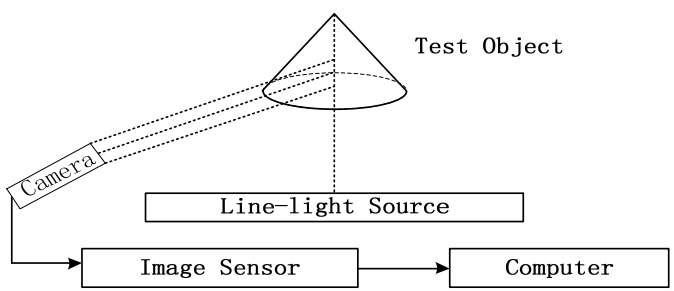

Fig. 1 Schematic diagram of system 
Model of Telecentric Camera. Bi-telecentric lens combines the advantages of object-side telecentric lenses and sensor-side telecentric lenses, and therefore the image magnification rate of the camera is constant when the object change in the range of the depth of field. In this case, the imaging model of telecentric camera established is:

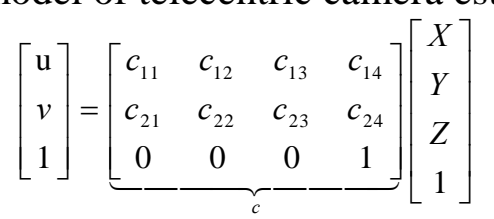

Where $(X, Y, Z)$ is world coordinate, and $(u, v)$ is coordinate of the pixel. All of the parameters in $c$ matrix can be calculated during calibration of the camera. Once the camera calibration is complete, the three-dimensional coordinate information of the object can be calculated from the mathematical model of the system with specific structure.

Measurement Model of Single-Camera. In this single-camera measurement model, three-dimensional coordinate information is obtained by two-dimensional image according to laser triangulation. When the light source and camera have been adjusted, the system can be set up as shown in Fig. 2. Respectively, two coordinate systems are established with origins from the camera optical center and the light source, whose three axes are parallel correspondingly. At the same time the camera's $x$-axis and the light source's $x$-axis coincide together, then the distance between the light source and the camera is $B$. The plane of the light source is always perpendicular to the horizontal plane.

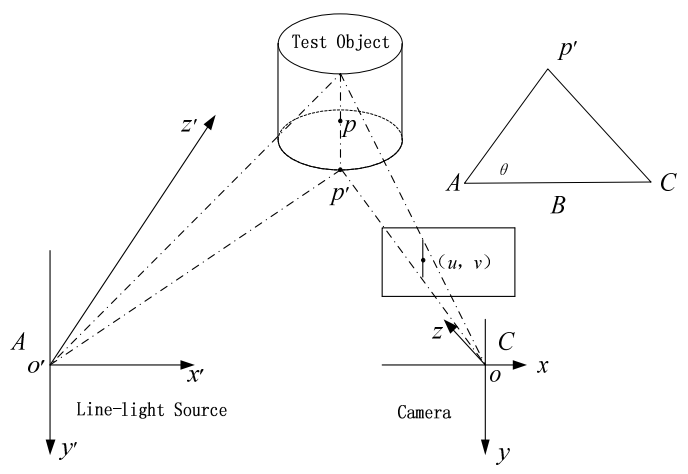

Fig. 2 single-camera model system

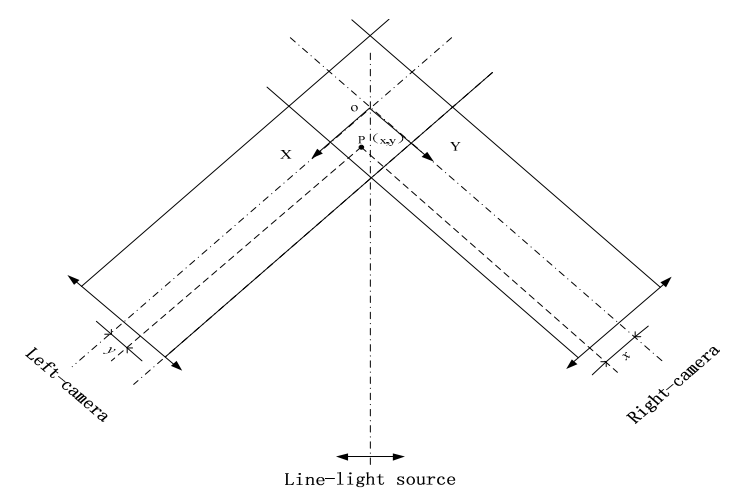

Fig.3 bis-camera model schematic diagram

If the imaging coordinate of a spatial point $p$ is $(u, v)$, according to the imaging principle, $x$ coordinate and $y$ coordinate can be calculated as follows:

$$
\left[\begin{array}{l}
\mathrm{u} \\
v
\end{array}\right]=\left[\begin{array}{ll}
\alpha & 0 \\
0 & \beta
\end{array}\right]\left[\begin{array}{l}
x \\
y
\end{array}\right]
$$

Where $\alpha, \beta$ are related to the internal and external parameters of camera, which can be obtained by the camera calibration. if $\theta$ is the angle between $x$-axis and the intersection line between the light plane and the horizontal, $z$ coordinates of the point can be calculated as follows:

$$
z=(x+B) \tan \theta
$$

So the three-dimensional coordinate information of the spatial point $p$ is calculated.

Measurement Model of Bis-Camera. To simplify the rotation matrix and translation matrix, as shown in Fig. 3, two cameras are vertically placed in bi-camera model, those optical axis coincide with two axes of the world coordinate system. The light source should be set in the middle of two cameras; meanwhile, the light stripe should be perpendicular to the horizontal. So the mathematical model of bi-camera can be organized in equations as follows:

$$
\begin{aligned}
& \left\{\begin{array}{l}
u^{L}=a^{L} Y_{w}+b^{L} Z_{w} \\
v^{L}=c^{L} Y_{w}+d^{L} Z_{w}
\end{array}\right. \\
& \left\{\begin{array}{l}
u^{R}=a^{R} X_{w}+b^{R} Z_{w} \\
v^{R}=c^{R} X_{w}+d^{R} Z_{w}
\end{array}\right.
\end{aligned}
$$


Where $a^{L}, a^{R}, b^{L}, b^{R}, c^{L}, c^{R}, d^{L}$ and $d^{R}$ can be calculated during calibration of the camera, a spatial point $p$ 's two-dimensional information can be calculated as $\left(Y_{w}, Z_{w}\right)$,according to the imaging coordinate $P_{L}\left(u^{L}, v^{L}\right)$. of left-side camera. In the same way, its $x$ coordinate and $z$ coordinate can be calculated as $\left(X_{w}, Z_{w}\right)$, by the right one. So the three-dimensional coordinate information $\left(X_{w}, Y_{w}, Z_{w}\right)$ is obtained.

System Software. Measurements need to figure out the target point in world coordinates according to the image pixel coordinates. It is extremely crucial for the three-dimensional measurement to extract the center-line of light with high accuracy. Before extracting center-line, the noise, mainly Salt \& Pepper noise and Gaussian noise, needs to be filtered out as much as possible to avoid affecting the measurement. In this paper, an adaptive median filter is selected, which not only has good effect to remove these noises, but also can be well preserving image detail. With regarding to the changeful characteristics of the image, an adaptive threshold segmentation method, which select the threshold by taking the entire image gray distribution, is adopted. There are many methods for center-line extraction, including Hessian matrix method, centroid method, extreme value method, the geometric center method, curve fitting method, etc. Among them, Hessian matrix method which has the highest precision is selected. Because the errors of this extraction will directly affect the accuracy of the three-dimensional coordinates, and then lead to reconstruct inaccurate dimensions of three-dimensional objects.

After the world coordinates of the point are obtained from the system of measurement model, it will be easy to figure out the point cloud data including three-dimensional contour of object. From geometrical terms, triangle is strongly available to describe complex geometric topology for its stability and flexibility. In this paper, Delaunay triangulation method is widely used to fit point cloud and reconstruct the outline of objects.

\section{Experimental Results}

A standard object, cylinder with $15.00 \mathrm{~mm}$ diameter, is to be measured and reconstructed in this paper to verify the stability and accuracy of the system, as shown in Fig.4(a). And its three- dimensional recovery map is emulated by matlab, according to the data measured, as shown in Fig.4(b).

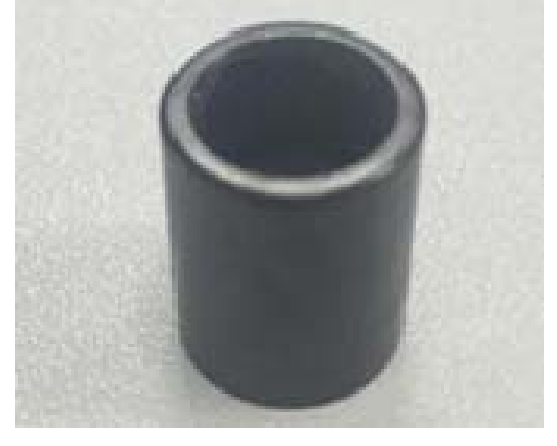

(a)

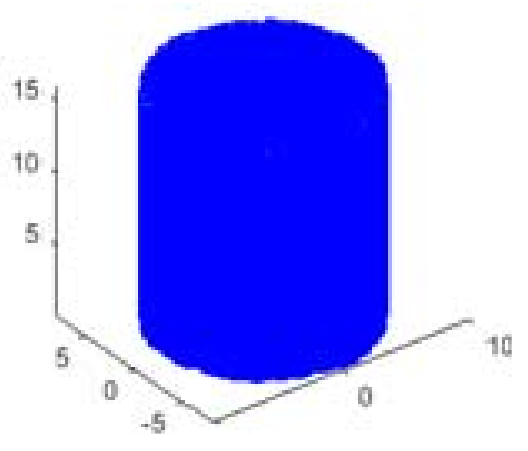

(b)

Fig. 4 (a)Testing object and (b)Recovery map of testing object

Camera calibration.Set the length of vernier caliper to $10 \mathrm{~mm}$, measure it 10 times and remove the large error data, at last the pixel value of a length of $10 \mathrm{~mm}$ obtained is 625.92 .

Rotation-axis calibration. Rotation-axis is calibrated by measuring the standard cylinder whose diameter is $4.79 \mathrm{~mm}$ when it's placed in ten groups of different places. Then remove the large error data, at last the pixel value of rotation-axis obtained is 600.05 .

Object measurement. the diameter of testing object is equal to the sum of two distances from two end-points of diameter to center of rotation-axis. The testing object is measured in per 5 degree to figure out its diameter. After one rotation, 20 couples of data is randomly selected as shown in Table 2. 
Table 1. Measured data of diameter

\begin{tabular}{cccccccc}
\hline No. & $\begin{array}{c}\text { Diameter } \\
{[\mathrm{mm}]}\end{array}$ & No. & $\begin{array}{c}\text { Diameter } \\
{[\mathrm{mm}]}\end{array}$ & No. & $\begin{array}{c}\text { Diameter } \\
{[\mathrm{mm}]}\end{array}$ & $\begin{array}{c}\text { Diameter } \\
{[\mathrm{mm}]}\end{array}$ \\
\hline 1 & 15.0635 & 6 & 14.9476 & 11 & 15.0685 & 16 & 15.0946 \\
2 & 15.0393 & 7 & 15.0536 & 12 & 15.0923 & 17 & 15.1675 \\
3 & 15.0542 & 8 & 15.0741 & 13 & 15.0601 & 18 & 14.9762 \\
4 & 15.1006 & 9 & 15.0532 & 14 & 14.9653 & 19 & 14.9572 \\
5 & 14.9728 & 10 & 14.9733 & 15 & 15.0288 & 20 & 15.0455
\end{tabular}

From Table 1, it's shown that the single measure deviation is within $0.1 \mathrm{~mm}$. The average of the data is $15.0506 \mathrm{~mm}$ and the standard deviation $0.0577 \mathrm{~mm}$. However, the 17 th data should be removed because its deviation is twice greater than standard deviation. So the diameter of testing object is measured as $15.0400 \mathrm{~mm}$.

\section{Conclusion}

In this paper, the author has mainly completed the establishment of measurement model based on bi-telecentric optic and experiment on the system building and verification. As the result shows, this measurement system can achieve high-precision three-dimensional measurement. While it is beyond no doubt that further research is still required to realize a more intelligent system and reconstruct the three-dimension of more complex shape objects.

\section{References}

[1] Cui Ximin, Ma Kaifeng, Huang Guiping. Research Progress and Development Trend Analysis of Modern Three-dimensional Industrial Measuring System[J]. Journal of North China University of Water Resources and Electric Power (Natural Science Edition). 2014,35(3): 56-57. (In Chinese)

[2] Y. Wang, S. Zhang. Superfast multifrequency phase-shifting technique with optimal pulse width modulation[J]. Optics Express,2011,19(16):5149-5155.

[3] Zhang Hu, Xiao Zhongkai, Fang Jianjun. A construction method of binocular vision system by three-dimensional coordinates[J]. Journal of Xi'an University of Arts \& Science: Nat. Sci. Ed., 2006,9(4):62-65. (In Chinese)

[4] Quan Yanming, Li Shumei, Mai Qingqun. On-machine 3D measurement of workpiece dimensions based on binocular vision[J]. Optics and Precision Engineering, 2013,21(4):1054-1061. (In Chinese)

[5] Li Guanyun. The State of the Art and Applications of the Industrial Measuring Systems[J]. Engineering of Surveying and Mapping. 2001, 10(2): 36-40. (In Chinese)

[6] Fang Ling. Research of Image Measurement Based on BI-TELECENTRIC System [D]. Guangzhou: South China University of Technology, 2013. (In Chinese)

[7] Li Jian, Chen Changming, Gao Wenuan. Research for the 3D information measuring technology [J]. Machinery Design and Manufacture .2010 (3): 222-224. (In Chinese)

[8] Liao Huiyang. Research on Precision Alignment of Optical Fibers Based on Binocular Stereo Micro-vision [D]. Guangzhou: South China University of Technology, 2014. (In Chinese)

[9] Hou, A. and Geng, Y. and Yuan, W.J. and Shi, F.G. The Implementation of Distance Measurement Using Binocular Vision[J]. Advanced Materials Research, 2012，490:711-715 